\title{
SOD2 and the mitochondrial UPR: partners regulating cellular phenotypic transitions
}

Chenxia He ${ }^{1,2,35}$, Peter C. Hart ${ }^{1,2,35}$, Doris Germain ${ }^{\S 4}$ and Marcelo G. Bonini ${ }^{1,2,35}$,

Departments of Medicine ${ }^{1}$, Pharmacology ${ }^{2}$, Pathology ${ }^{3}$, College of Medicine of the University of Illinois at Chicago, Chicago, IL, 60612, USA and ${ }^{4}$ Department of Medicine (Hematology and Medical Oncology) Icahn School of Medicine at Mount Sinai, New York, NY, 10029, USA

\section{${ }^{8}$ Correspondence to:}

Marcelo G. Bonini, Ph.D., Departments of Medicine, Pathology and Pharmacology, College of Medicine at the University of Illinois at Chicago, UIC, 909 S. Wolcott Ave, COMRB 1131,Chicago, IL, 60612, Phone: (312) 355-5948; email: mbonini@uic.edu 


\section{ABSTRACT}

ATP and reactive oxygen species (ROS) are signaling molecules that control cellular function and phenotype. Mitochondria produce both ATP and ROS. Since the electrons needed to generate either ATP or ROS originate from $\mathrm{NADH} / \mathrm{FADH}$, the mechanism through which electrons flow towards oxygen determines yields and whether ATP or ROS prevail. Alterations in the electron flow impact cells dramatically, such as by supporting specialization (which requires high ATP) or imposing dedifferentiation. High $\mathrm{ROS}$, facilitated by enzymes such as SOD2 that enhance mitochondrial $\mathrm{H}_{2} \mathrm{O}_{2}\left(\mathrm{mtH}_{2} \mathrm{O}_{2}\right)$, are normally linked to dedifferentiation of somatic cells. Here, we propose that combined, high $\mathrm{mtH}_{2} \mathrm{O}_{2}$ and mitochondrial UPR (UPR ${ }^{\mathrm{mt}}$ ) activation are essential for somatic dedifferentiation programs and the acquisition of stem-like properties in reparative processes and disease. (120 words)

\section{Mitochondrial electronics in cellular fate determination}

Mitochondria are now recognized as central players regulating cell signaling at both a cellular ${ }^{[1-3]}$ and organismal leve[ ${ }^{[4]}$ via mitochondria-encoded hormones ${ }^{[5,6]}$. Superoxide radicals and their unavoidable product hydrogen peroxide $\left(\mathrm{H}_{2} \mathrm{O}_{2}\right)$, commonly referred to as reactive oxygen species (ROS), are two examples of short lived reactive metabolites of oxygen that directly impact the activity of a large number of enzyme families including thiol phosphatases ${ }^{[7,8]}$ and caspases $^{[9]}$. Indirectly, they also impact kinase ${ }^{[10-13]}$, the proteasome ${ }^{[14]}$ and transcription factor activity and function [15-17]. Therefore, mitochondrial ROS (mtROS) are direct effectors of signaling, and enzymes controlling their formation and metabolism serve as key regulators of cellular signaling networks". Along with ROS, ATP--another major product of mitochondria--has also been implicated in the regulation of cell signaling events and is required for the maintenance of cellular differentiation and specialization. This article discusses the roles of ATP and $\mathrm{H}_{2} \mathrm{O}_{2}$ as switch mechanisms controlling transitions between cellular phenotypes divided arbitrarily into two major states: differentiated (which demands high mitochondrial ATP (mtATP) production $^{[18-20]}$ ) and undifferentiated (which has less or minimal dependence on mitochondrial ATP $^{[21-23]}$ and that are normally promoted by high ROS). Since the degree of dedifferentiation each cell type seems to tolerate is different, we take "undifferentiated" as a term to refer to phenotypes that are less differentiated than the parent cell either because of the appearance of molecular markers or the acquisition of functional traits that characterize stemness. Our goal is to explore a different view centered on the conveniences of cells that are trying to survive stress and for which specialization is a dispensable luxury that is maintained at the expense of a high ATP demand. According to this perspective, the lower ATP states imposed by reduced mitochondrial bioenergetic activity forces cells to give up specialization and/or differentiation and instead utilize the available energy to maintain the basic functions needed for survival. From the standpoint of the multicellular organism, cell survival at the cost of loss of specialization and function equals disease whereby insufficient performance of a particular cell type compromises the well-being and operation of the whole system. Therefore, we propose that mitochondria (via their production of ATP and ROS, particularly the diffusible $\mathrm{H}_{2} \mathrm{O}_{2}$ ) direct cellular plasticity by enabling differentiation or promoting dedifferentiation (Figure 1). Normally, transient dedifferentiation is a fundamental physiologic process that allows organisms to exist in environments that constantly change by conferring cells with the plasticity needed for tissues to adapt, remodel and repair. However, subversion of the fine balance between mtATP and 
mtROS production with chronic loss of cellular function through dedifferentiation can evolve to a scenario permissive to disease that ranges from tissue degeneration and loss of function to metabolic syndrome and cancer.

\section{ATP and $\mathrm{H}_{2} \mathrm{O}_{2}$ as constituents of a mitochondria switch mechanism activating phenotypic transitions}

Not only are ATP and $\mathrm{H}_{2} \mathrm{O}_{2}$ two of the most important products of mitochondrial activity, but the rate and relative abundance of their production represents the combined functional outcome of every single subtle change impacting how electrons flow through mitochondria towards $\mathrm{O}_{2}$. This is demonstrated by the observation that inhibitors of the electron transport chain, which cause an enrichment in reduced electron carriers, divert ATP synthesis to ROS formation ${ }^{[24,25]}$. It is also exemplified by the observation that accumulated tricarboxylic acid cycle (TCA) metabolites, that are processed in mitochondria, increase ROS normally because of an overflow of electrons from complex II. This overflow of electrons from complex II powers both ATP synthesis (via transfer of these electrons forward to complexes III and IV) and ROS (via reverse flow of electrons back to complex I) ${ }^{[26]}$. Hence, interference with the delicate and tightly regulated interactions of the electron transport chain (ETC) complexes with their electron carriers (and each other) normally results in disruption of ATP synthesis and increased ROS production impacting metabolic and signaling networks at a cellular level, as discussed in further detail later.

\section{Differentiation as a cellular luxury: The roles of ATP in survival and specialization}

Most of the available studies appear to agree that ATP produced from mitochondria is dispensable for cell survival since it is also produced by alternative pathways such as glycolysis and amino acid breakdown. However, mitochondrial ATP is required to support the activities of specialized (differentiated) cells, which demand relatively high ATP levels that are not attainable independent of mitochondria. Glycolysis alone produces 2 net (4 in total) ATP molecules. When combined with the oxidation of glycolytic product pyruvate by mitochondria, 36 molecules of ATP are obtained. This level of ATP is needed to support specialized cellular functions such as the maintenance of the rhythm and contractility of heart cells, the polarization of neural cells, the high metabolic activity of cells that carry out biosynthetic functions synthetic cell along with the active uptake and pumping of different metabolites across the membranes of various polarized and secretory cells. In this regard the luxury of specialization imposes energetic requirements that can only be met via ATP production by mitochondria, indicating that mitochondrial respiration may actually be a prerequisite of differentiation.

\section{What do mitochondria do when not making ATP?}

Mounting evidence supports a role for mitochondria-derived ROS in cell signaling, making ROS an essential bioactive product of mitochondria. Although the term "ROS" encompasses a number of species, ROS produced by mitochondria include superoxide radicals (which act either as weak oxidants or powerful reductants depending on the target and do not diffuse through membranes) ${ }^{[27]}$ and $\mathrm{H}_{2} \mathrm{O}_{2}$ (a powerful oxidant that diffuses through membranes). $\mathrm{H}_{2} \mathrm{O}_{2}$ is a strong oxidant prone to react with thiols and methionine residues at relatively high rates $^{[28]}$. 
Oxidation of active thiol residues on phosphatases inactivates them promoting the unopposed action of kinases. This illustrates the critical role of $\mathrm{H}_{2} \mathrm{O}_{2}$ in functioning as a direct regulator of cell signaling and positions mitochondria as central orchestrators dampening, activating, amplifying and nullifying signals initiated by receptors, intracellular calcium level variations, second messengers and intracellular enzymes. Energetically it appears that increases in mitochondrial ROS coincide with a decrease in ATP, which is expected since both ATP and ROS production require electrons from reduced cofactors $\mathrm{NADH} / \mathrm{FADH} \mathrm{H}_{2}$ and, in addition to these, $\mathrm{O}_{2}$ as the electron acceptor. Curiously, although undifferentiated, progenitor, stem and cancer cells have drastically different energetic needs, all appear to rely on glycolysis to meet their energetic demands ${ }^{[18,20,29,30]}$. Mitochondria in these cells are not inactive and their roles may be related to the maintenance of undifferentiated phenotypes common to most of these cell types.

The exact mechanisms by which mitochondria or even heterogeneous mitochondrial subpopulations within a cell operate these phenotypic changes are still incompletely understood, although there is enough evidence indicating an essential role for mitochondrial ROS. One possibility is that ROS may subvert the hypoxia-induced factor system, which normally responds to oxygen deprivation, thereby simulating hypoxia at the cellular level even when oxygen is plentiful abundance (Box 1). Hypoxia induced factor (HIF)a subunits, the transcriptionally active core units of hypoxia induced factors, are anomalously activated by elevated $\left.\mathrm{H}_{2} \mathrm{O}_{2}{ }^{[31}, 32\right]$, indicating that a diversion in mitochondrial activity towards enhancing $\mathrm{H}_{2} \mathrm{O}_{2}$ levels could rewire transcription via HIF activation. Other transcriptional effectors activated by ROS include Nrf2 ${ }^{[33,34]}$ and NFK $\mathbf{B}^{[17,35,36]}$, which further exemplify how changes in ROS steady-

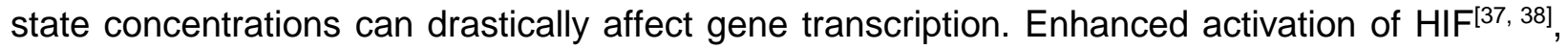
Nrf2 and $\mathrm{NF}_{\kappa} \mathrm{B}^{[39-41]}$ signaling maintains, directs and in some cases imposes (un)differentiation. For instance, Nrf2 has been implicated in the regulation of self-renewal and the maintenance of pluripotency in stem cells ${ }^{[42]}$. Therefore, a functional switch established by the balance between ATP and ROS production in mitochondria seems to be emerging from recent studies.

Based on the rationale that mitochondria direct phenotypic transitions via ROS and ATP, we propose that at least two functional states exist: state I (where ATP production prevails over ROS) and state II (where ROS production prevails over ATP, Figure 2). Normally, when ATP production prevails, mitochondria are referred to as functional, fit and young. Mitochondria producing high ROS levels are referred to as dysfunctional and old. A recent paper from the Sabatini group ${ }^{[4]}$ showed that during asymmetric division stem-like cells recognize and segregate mitochondria in accordance to their age gifting mixed pools of mitochondria to both daughter cells. In the same study, it was found that although daughter cells more likely to differentiate received higher numbers of "old" mitochondria though both daughter cells retained a fraction of young and old organelles. The fact that some "old" mitochondria remained in the stem cell like pool is puzzling and difficult to reconcile with the idea that highest quality organelles should be dedicated to preserving the stem cell pool. However, it is more in line with a different view that perhaps $\mathrm{H}_{2} \mathrm{O}_{2}$ production by mitochondria per se is not a manifestation of dysfunction but a mere distinction of states functionally operating different programs based on the energetic and signaling needs of the differentiating versus non-differentiating cells. From these observations and reflections a central question emerges: What is/are the switch/es controlling transitions between state I and state II, and how do they regulate mitochondria to 
broadly affect the cellular signaling network? Although a more complete answer to this question requires further studies, enzymes that regulate the formation of superoxide radicals and their conversion to $\mathrm{H}_{2} \mathrm{O}_{2}$ in mitochondria are at a first glance likely to play a role.

\section{MnSOD/SOD2 as a key effector of mitochondrial ROS metabolism}

SOD2 is one of the three identified SOD enzymes and the only one that binds manganese in mammalian cells. Distinct from its congeners, SOD2 resides predominantly in the mitochondrial matrix and is widely accepted to work primarily as a catalyst of superoxide radical dismutation to $\mathrm{H}_{2} \mathrm{O}_{2}$ (and oxygen). In contrast to superoxide radicals (which bear a net negative charge and are thus impermeable to membranes), $\mathrm{H}_{2} \mathrm{O}_{2}$ is neutral, highly soluble in water and freely diffuses throughout the cell and beyond. Another important difference is that superoxide radicals are actually reductants serving in most cases as electrons donors, while $\mathrm{H}_{2} \mathrm{O}_{2}$ is an oxidant prone to abstract electrons from nucleophilic molecules.

Since superoxide radicals dismutate spontaneously, it may seem counter-intuitive that a dismutase is even needed to ensure the production of $\mathrm{H}_{2} \mathrm{O}_{2}$ from superoxide radicals in mitochondria. However, there are multiple redox active components in the mitochondria that can accept electronsredox environment of mitochondria is highly active suggesting that there are numerous potential sinks for superoxide radicalslimiting its dismutation to $\mathrm{H}_{2} \mathrm{O}_{2}$ and $\mathrm{O}_{2}$. These include cytochromes, redox active metal centers and multiple non-metallic electron acceptors) that eliminate superoxide anions without the concomitant production of $\mathrm{H}_{2} \mathrm{O}_{2}$. Hence, it is more than likely that these electron carriers may work as natural impediments for the efficient production of $\mathrm{H}_{2} \mathrm{O}_{2}$ from the spontaneous dismutation of superoxide, thereby imposing the need of SOD2 to efficiently turn superoxide radicals into $\mathrm{H}_{2} \mathrm{O}_{2}$, although studies proving this are still missing. This competition model (depicted in Figure 3 ) is consistent with the findings that higher SOD2 levels translate into higher $\mathrm{H}_{2} \mathrm{O}_{2}{ }^{[7,12]}$ although the increased $\mathrm{H}_{2} \mathrm{O}_{2}$ cannot be simply explained by the catalyzed dismutation of superoxide radicals ${ }^{[44]}$. Thus, we suggest that the activity of SOD2 in mitochondria is required both for the formation of $\mathrm{H}_{2} \mathrm{O}_{2}$ and for its signaling in cells that are addicted to operating at a higher redox potential (e.g. cancer cells). For instance, it has been demonstrated that the elevation of SOD2-derived $\mathrm{H}_{2} \mathrm{O}_{2}$ in breast cancer cells is essential for metabolic adaptation ${ }^{[11,12]}$. Therefore, it appears that SOD2 is a key source of redox signals generated in mitochondria to direct various redox sensitive processes in the cell. How cells adapt to survive high SOD2/high $\mathrm{H}_{2} \mathrm{O}_{2}$ is an important question, since it appears that concomitant enhanced $\mathrm{H}_{2} \mathrm{O}_{2}$ formation and activation of mechanisms that confer $\mathrm{H}_{2} \mathrm{O}_{2}$ resistance supports multiple cellular phenotypes, some of which are pathologic or oncogenic and are characterized by hyperproliferation and poor differentiation (Box 2).

\section{The UPR ${ }^{\mathrm{mt}}$ as a quality control system enabling state II.}

If SOD2 regulates the switch between state I and state II via acting as a source of $\mathrm{H}_{2} \mathrm{O}_{2}$, and is selectively up-regulated in undifferentiated cells such as progenitor and stem cells, this observation raises fundamental questions such as (i) what regulates SOD2 expression and function in these cells(ii) what is the selective advantage of increasing $\mathrm{H}_{2} \mathrm{O}_{2}$ and (iii) how cells adapt to resist high ROS levels. We hypothesize that the mitochondrial unfolded protein response (UPR ${ }^{m t}$ ) is the mechanism by which SOD2 is up-regulated, and that in addition to 
SOD2, the activation of the large and complex transcriptional program regulated by the UPR ${ }^{\mathrm{mt}}$ results in increased mitochondrial fitness and the ability to buffer ROS in these cells (Figure 4).

The UPR ${ }^{m t}$ was first described by the Hoogenraad group, who reported that the transcription factor CHOP is necessary for elevation in the transcription of hsp60, hsp10 and the protease ClpPin response to the accumulation of misfolded proteins in the matrix ${ }^{[45]}$. Somehow, the role of $\mathrm{CHOP}$ in the UPR ${ }^{\mathrm{mt}}$ has since been forgotten. Rather, following this initial study, others have dissected the UPR ${ }^{\mathrm{mt}}$ in Caenorhabditis elegans (C. elegans) using the promoter of hsp60 as a reporter for the activation of this response. These studies have focused on the transcription factor ATFS1, for which no mammalian homologue has been identified yet ${ }^{[46-48]}$. In addition, the use of a single reporter has lead to the misconception that activation of the transcription of chaperones defines the UPR ${ }^{\mathrm{mt}}$. For example, because the retrograde response (RTG) that was described in yeast in response to decreased mitochondrial activity by the Butow group ${ }^{[49]}$ does not lead to the transcription of chaperones, the RTG is considered distinct from the UPR ${ }^{\mathrm{mt}}\left[{ }^{[50]}\right.$. However, a genome-wide RNAi screen revealed that several negative regulators of the UPR ${ }^{\mathrm{mt}}$ do not activate the hsp-60 reporter, therefore further supporting this concern [51]. This misconception has wider impact since, for instance, ROS is reported to activate the retrograde response but not the UPR ${ }^{\mathrm{mt}}$, again as defined by activation of the hsp60-reporter. Yet, a recent study reported that ROS generated as a result of increased NAD+ levels using PARP inhibitors activates UPR ${ }^{\mathrm{mt}}\left[{ }^{[52]}\right.$. Importantly for this review, SOD2 was found to be a key player of the UPR ${ }^{\mathrm{mt}}$ in this study. Likewise, it was reported that SOD2 expression is activated by the UPR ${ }^{\mathrm{mt}}{ }^{[53]}$. Further, we found that inhibition of CHOP, which completely abolished the activation of hsp60 by accumulation of misfolded proteins in the mitochondria in mammalian cells, had no effect on the activation of SOD2 ${ }^{[53]}$. Rather, the expression of SOD2 was dependent on the transcription factor FOXO3a, which was itself dependent on the activity of the mitochondrial sirtuin (SIRT) SIRT3 ${ }^{[53]}$.

How SIRT3 in the mitochondria can affect the deacetylation of FOXO3a in the cytoplasm is unclear; however, SIRT3 activation of FOXO3a has been reported by several groups. For instance, FOXO3a occupancy of the SOD2 promoter was reported to be decreased in Sirt3 ${ }^{-/}$ mouse embryonic fibroblasts due to a decrease in the nuclear accumulation of FOXO3a. Additionally, the presence of a dominant negative construct of SIRT3 reduced the level of FOXO3a, suggesting that SIRT3 somehow affects the nuclear accumulation of FOXO3a ${ }^{[54]}$. Collectively, these findings indicate that the UPR ${ }^{\mathrm{mt}}$ is composed of several axes. The CHOPdependent axis is responsible for promoting the expression of mitochondrial chaperones and proteases, while the SIRT3 axis stimulates the expression of the mitochondrial stress resistance machinery. We also reported that several markers of mitophagy are up-regulated under mitochondrial proteotoxic conditions ${ }^{[53]}$. As for the expression of SOD2, mitophagy is dependent on SIRT3 but was independent of CHOP. Therefore, the machineries that limit oxidative damage and mitophagy are both regulated by the SIRT3 axis of the UPR ${ }^{\mathrm{mt}}$. These observations suggest that SIRT3 acts as a mitochondrial checkpoint protein, whereby moderately stressed mitochondria may be repaired through an up-regulation of the stress resistance machinery, while irreversibly damaged organelles are selectively eliminated from the network by mitophagy. This possibility is consistent with the finding that mitochondrial proteotoxic stress leads to the accumulation of PINK and the translocation of the ubiquitin ligase PARK2 to the irreversibly 
damaged mitochondria ${ }^{[55]}$. The complexity of the $U P R^{m t}$ is reflected by the fact that mitochondrial proteotoxic stress also induces the expression of the transcription factor nuclear respiratory factor 1 (NRF-1) ${ }^{[53,56]}$ Along with PGC-1 $\alpha$, NRF-1 is a major regulator of mitochondrial biogenesis. These findings indicate that the UPR ${ }^{\text {mt }}$ represents a ballet of wellchoreographed signaling pathways that collectively maintain the integrity of the entire network. The UPR ${ }^{\text {mt }}$ does this by balancing the elimination of irreversibly damaged mitochondria with the biogenesis of new organelles and the repair of pre-existing mitochondria. This is accomplished via the activation of antioxidant machinery, including SOD2, but also requires the expression of enzymes that detoxify the product of SOD2 $\left(\mathrm{H}_{2} \mathrm{O}_{2}\right)$ and the expression of chaperones that oppose $\mathrm{H}_{2} \mathrm{O}_{2}$-induced damage Therefore, the activation of not only SOD2 but also the entire $U_{P R}{ }^{m t}$ may represent a powerful mechanism to maintain the integrity of the mitochondrial network in undifferentiated cells such as stem cells.

This possibility is supported by two recent reports. First, the activity of FOXO3a was found to be required for hematopoietic stem cell (HSC) homeostasis. While these cells are dormant, they maintain the potential to be activated to reconstitute blood. This re-activation requires a switch from glycolysis $\left(\mathrm{H}_{2} \mathrm{O}_{2}\right.$, state $\left.\mathrm{II}\right)$ to mitochondrial oxidative phosphorylation (ATP, State I). Maintenance of low superoxide is necessary for sustaining HSC dormancy while we contend that elevated $\mathrm{H}_{2} \mathrm{O}_{2}$ promotes and/or enhances signaling that maintains stemness (e.g. hypoxia-induced factors). FOXO3 was found to be required to keep the levels of superoxide low, and the absence of FOXO3a in HSC caused impaired mitochondrial metabolism [57]. Second, the activity of NRF1 was reported to be linked to the UPR ${ }^{\mathrm{mt}}$ and the maintenance of the regenerative capacity of HSCs ${ }^{[58]}$. In this axis of the UPR ${ }^{\mathrm{mt}}$, the authors further describe that yet another sirtuin, SIRT7, is implicated. SIRT7 is a histone deacetylase that has been identified to directly bind NRF1 ${ }^{[58]}$. Further, NRF1 regulates the binding of SIRT7 to specific targets such as the promoters of mitochondrial ribosomal protein and translation factors to mediate their transcriptional repression [58].

In summary, the transcriptional program activated by the UPR ${ }^{\mathrm{mt}}$ is likely to be responsible for the up-regulation of SOD2. Further, the UPR ${ }^{m t}$ may explain the recent counterintuitive finding from the Sabatini group that during asymmetric division the renewing stem cells retain mitochondria with higher $\mathrm{H}_{2} \mathrm{O}_{2}$, which may be essential for the activation of the $\mathrm{UPR}^{\mathrm{mt}}$.

\section{Concluding remarks: SIRT3, SOD2 and the UPR ${ }^{\mathrm{mt}}$, a functional triad in mitochondria- driven signaling}

From an organismal perspective the ability of cells to acquire functional phenotypes and to adequately adjust to changes in the metabolic, hormonal and physical state of the host maintains health. Although some phenotypic/functional flexibility seems to be required to promote adaptation and repair it is becoming increasingly clearer that the subversion of mechanisms of phenotypic plasticity leads to disease states characterized by highly resilient and well adapted cells. Cancer and fibrotic disease seem to be examples of conditions fitting this definition. Despite many open-ended questions, it is becoming clear that the switch between ATP and $\mathrm{H}_{2} \mathrm{O}_{2}$ production in mitochondria is a determinant of cellular fate decisions. Many 
regulators of this switch are still to be identified, but from available studies it appears that SOD2 and the UPR ${ }^{\mathrm{mt}}$ combined enable the existence of cells that can adapt to survive under high $\mathrm{H}_{2} \mathrm{O}_{2}$ conditions. The UPR ${ }^{\mathrm{mt}}$ is critical to promote mitochondria quality control when the organelles are operating at high $\mathrm{H}_{2} \mathrm{O}_{2}$ producing efficiency. Thus, the duo SOD2/UPR ${ }^{\mathrm{mt}}$ appears to regulate aspects of the mitochondria-nuclear communication essential for the acquisition of phenotypic plasticity and resilience in response to metabolic stress. In isolation SOD2 is a source of $\mathrm{mtH}_{2} \mathrm{O}_{2}$. When combined with the capacity to buffer $\mathrm{H}_{2} \mathrm{O}_{2}$ activity (e.g. via activation of the UPR ${ }^{\mathrm{mt}}$ ) SOD2 becomes a regulator of signaling in the cell. Therefore, we contend that phenotypically plastic cells can be identified by the simultaneous activation of $\mathrm{SOD} 2, \mathrm{mtH}_{2} \mathrm{O}_{2}$, and the UPR ${ }^{\mathrm{mt}}$, although the nuances distinguishing physiologic from pathogenic activation are yet to be determined.

\section{ACKNOWLEDGEMENTS}

The authors would like to acknowledge the following agencies for providing funds that support research in their laboratories. U.S. Department of Defense (grant W911NF-15-1-0410 to M.G.B.); National Heart Lung and Blood Institute (grant 2RO1HL125356 to M.G.B.(MPI)); and National Cancer Institute (grant 1R01CA172046-01A1 to D.G.).

\section{REFERENCES}

1. Shaughnessy DT, McAllister K, Worth L, Haugen AC, Meyer JN, Domann FE, et al. Mitochondria, energetics, epigenetics, and cellular responses to stress. Environ Health Perspect. 2014;122(12):1271-8. doi: 10.1289/ehp.1408418. PubMed PMID: 25127496; PubMed Central PMCID: PMCPMC4256704.

2. Chandel NS. Evolution of Mitochondria as Signaling Organelles. Cell Metab. 2015;22(2):204-6. doi: 10.1016/j.cmet.2015.05.013. PubMed PMID: 26073494.

3. Simon MC. Coming up for air: HIF-1 and mitochondrial oxygen consumption. Cell Metab. 2006;3(3):150-1. doi: 10.1016/j.cmet.2006.02.007. PubMed PMID: 16517402.

4. Shadel GS, Horvath TL. Mitochondrial ROS Signaling in Organismal Homeostasis. Cell. 2015;163(3):560-9. doi: 10.1016/j.cell.2015.10.001. PubMed PMID: 26496603; PubMed Central PMCID: PMCPMC4634671.

5. Lee C, Zeng J, Drew BG, Sallam T, Martin-Montalvo A, Wan J, et al. The mitochondrial-derived peptide MOTS-c promotes metabolic homeostasis and reduces obesity and insulin resistance. Cell Metab. 2015;21(3):443-54. doi: 10.1016/j.cmet.2015.02.009. PubMed PMID: 25738459; PubMed Central PMCID: PMCPMC4350682.

6. Guo B, Zhai D, Cabezas E, Welsh K, Nouraini S, Satterthwait AC, et al. Humanin peptide suppresses apoptosis by interfering with Bax activation. Nature. 2003;423(6938):456-61. doi: 10.1038/nature01627. PubMed PMID: 12732850.

7. Connor KM, Subbaram S, Regan KJ, Nelson KK, Mazurkiewicz JE, Bartholomew PJ, et al. Mitochondrial $\mathrm{H} 2 \mathrm{O} 2$ regulates the angiogenic phenotype via PTEN oxidation. J Biol Chem. 2005;280(17):16916-24. doi: 10.1074/jbc.M410690200. PubMed PMID: 15701646. 
8. Lee SR, Kwon KS, Kim SR, Rhee SG. Reversible inactivation of protein-tyrosine phosphatase 1B in A431 cells stimulated with epidermal growth factor. J Biol Chem. 1998;273(25):15366-72. PubMed PMID: 9624118.

9. Borutaite V, Brown GC. Caspases are reversibly inactivated by hydrogen peroxide. FEBS Lett. 2001;500(3):114-8. PubMed PMID: 11445067.

10. He BJ, Joiner ML, Singh MV, Luczak ED, Swaminathan PD, Koval OM, et al. Oxidation of CaMKII determines the cardiotoxic effects of aldosterone. Nat Med. 2011;17(12):1610-8. doi: 10.1038/nm.2506. PubMed PMID: 22081025; PubMed Central PMCID: PMCPMC3332099.

11. Hart PC, Ratti BA, Mao M, Ansenberger-Fricano K, Shajahan-Haq AN, Tyner AL, et al. Caveolin-1 regulates cancer cell metabolism via scavenging $\mathrm{Nrf2}$ and suppressing MnSOD-driven glycolysis. Oncotarget. 2015. doi: 10.18632/oncotarget.5687. PubMed PMID: 26543228.

12. Hart PC, Mao M, de Abreu AL, Ansenberger-Fricano K, Ekoue DN, Ganini D, et al. MnSOD upregulation sustains the Warburg effect via mitochondrial ROS and AMPK-dependent signalling in cancer. Nat Commun. 2015;6:6053. doi: 10.1038/ncomms7053. PubMed PMID: 25651975; PubMed Central PMCID: PMCPMC4319569.

13. Sun $Y$, Hu G, Zhang $X$, Minshall RD. Phosphorylation of caveolin-1 regulates oxidant-induced pulmonary vascular permeability via paracellular and transcellular pathways. Circ Res. 2009;105(7):67685, 15 p following 85. doi: 10.1161/CIRCRESAHA.109.201673. PubMed PMID: 19713536; PubMed Central PMCID: PMCPMC2776728.

14. Demasi M, Silva GM, Netto LE. 20 S proteasome from Saccharomyces cerevisiae is responsive to redox modifications and is S-glutathionylated. The Journal of biological chemistry. 2003;278(1):679-85. Epub 2002/11/01. doi: 10.1074/jbc.M209282200. PubMed PMID: 12409293.

15. Wilson LA, Gemin A, Espiritu R, Singh G. ets-1 is transcriptionally up-regulated by $\mathrm{H} 2 \mathrm{O} 2$ via an antioxidant response element. FASEB journal : official publication of the Federation of American Societies for Experimental Biology. 2005;19(14):2085-7. doi: 10.1096/fj.05-4401fje. PubMed PMID: 16234432.

16. Huang LE, Arany Z, Livingston DM, Bunn HF. Activation of hypoxia-inducible transcription factor depends primarily upon redox-sensitive stabilization of its alpha subunit. The Journal of biological chemistry. 1996;271(50):32253-9. PubMed PMID: 8943284.

17. Zhang J, Johnston G, Stebler B, Keller ET. Hydrogen peroxide activates NFkappaB and the interleukin-6 promoter through NFkappaB-inducing kinase. Antioxid Redox Signal. 2001;3(3):493-504. doi: 10.1089/15230860152409121. PubMed PMID: 11491660.

18. De Bock K, Georgiadou M, Schoors S, Kuchnio A, Wong BW, Cantelmo AR, et al. Role of PFKFB3driven glycolysis in vessel sprouting. Cell. 2013;154(3):651-63. doi: 10.1016/j.cell.2013.06.037. PubMed PMID: 23911327.

19. Kuppusamy KT, Jones DC, Sperber H, Madan A, Fischer KA, Rodriguez ML, et al. Let-7 family of microRNA is required for maturation and adult-like metabolism in stem cell-derived cardiomyocytes. Proc Natl Acad Sci U S A. 2015;112(21):E2785-94. doi: 10.1073/pnas.1424042112. PubMed PMID: 25964336; PubMed Central PMCID: PMCPMC4450404.

20. Sperber H, Mathieu J, Wang Y, Ferreccio A, Hesson J, Xu Z, et al. The metabolome regulates the epigenetic landscape during naive-to-primed human embryonic stem cell transition. Nat Cell Biol. 2015;17(12):1523-35. doi: 10.1038/ncb3264. PubMed PMID: 26571212; PubMed Central PMCID: PMCPMC4662931.

21. Folmes CD, Nelson TJ, Martinez-Fernandez A, Arrell DK, Lindor JZ, Dzeja PP, et al. Somatic oxidative bioenergetics transitions into pluripotency-dependent glycolysis to facilitate nuclear reprogramming. Cell Metab. 2011;14(2):264-71. doi: 10.1016/j.cmet.2011.06.011. PubMed PMID: 21803296; PubMed Central PMCID: PMCPMC3156138. 
22. Panopoulos AD, Yanes O, Ruiz S, Kida YS, Diep D, Tautenhahn R, et al. The metabolome of induced pluripotent stem cells reveals metabolic changes occurring in somatic cell reprogramming. Cell Res. 2012;22(1):168-77. doi: 10.1038/cr.2011.177. PubMed PMID: 22064701; PubMed Central PMCID: PMCPMC3252494.

23. Zhang J, Khvorostov I, Hong JS, Oktay Y, Vergnes L, Nuebel E, et al. UCP2 regulates energy metabolism and differentiation potential of human pluripotent stem cells. EMBO J. 2011;30(24):486073. doi: 10.1038/emboj.2011.401. PubMed PMID: 22085932; PubMed Central PMCID: PMCPMC3243621.

24. Boveris A, Cadenas E. Mitochondrial production of superoxide anions and its relationship to the antimycin insensitive respiration. FEBS letters. 1975;54(3):311-4. Epub 1975/07/01. PubMed PMID: 236930.

25. Cadenas $\mathrm{E}$, Boveris $\mathrm{A}$, Ragan $\mathrm{Cl}$, Stoppani AO. Production of superoxide radicals and hydrogen peroxide by $\mathrm{NADH}$-ubiquinone reductase and ubiquinol-cytochrome c reductase from beef-heart mitochondria. Archives of biochemistry and biophysics. 1977;180(2):248-57. Epub 1977/04/30. PubMed PMID: 195520.

26. Chouchani ET, Pell VR, Gaude E, Aksentijevic D, Sundier SY, Robb EL, et al. Ischaemic accumulation of succinate controls reperfusion injury through mitochondrial ROS. Nature. 2014;515(7527):431-5. Epub 2014/11/11. doi: 10.1038/nature13909. PubMed PMID: 25383517; PubMed Central PMCID: PMC4255242.

27. Buettner GR. The pecking order of free radicals and antioxidants: lipid peroxidation, alphatocopherol, and ascorbate. Archives of biochemistry and biophysics. 1993;300(2):535-43. Epub 1993/02/01. doi: 10.1006/abbi.1993.1074. PubMed PMID: 8434935.

28. Bonini MG, Consolaro ME, Hart PC, Mao M, de Abreu AL, Master AM. Redox control of enzymatic functions: The electronics of life's circuitry. IUBMB Life. 2014. doi: 10.1002/iub.1258. PubMed PMID: 24668617.

29. Ochocki JD, Simon MC. Nutrient-sensing pathways and metabolic regulation in stem cells. The Journal of cell biology. 2013;203(1):23-33. Epub 2013/10/16. doi: 10.1083/jcb.201303110. PubMed PMID: 24127214; PubMed Central PMCID: PMC3798256.

30. DeNicola GM, Cantley LC. Cancer's Fuel Choice: New Flavors for a Picky Eater. Molecular cell. 2015;60(4):514-23. Epub 2015/11/23. doi: 10.1016/j.molcel.2015.10.018. PubMed PMID: 26590711; PubMed Central PMCID: PMC4676726.

31. Chandel NS, Maltepe E, Goldwasser E, Mathieu CE, Simon MC, Schumacker PT. Mitochondrial reactive oxygen species trigger hypoxia-induced transcription. Proceedings of the National Academy of Sciences of the United States of America. 1998;95(20):11715-20. Epub 1998/09/30. PubMed PMID: 9751731; PubMed Central PMCID: PMC21706.

32. Brunelle JK, Bell EL, Quesada NM, Vercauteren K, Tiranti V, Zeviani M, et al. Oxygen sensing requires mitochondrial ROS but not oxidative phosphorylation. Cell metabolism. 2005;1(6):409-14. Epub 2005/08/02. doi: 10.1016/j.cmet.2005.05.002. PubMed PMID: 16054090.

33. Ding Y, Choi KJ, Kim JH, Han X, Piao Y, Jeong JH, et al. Endogenous hydrogen peroxide regulates glutathione redox via nuclear factor erythroid 2-related factor 2 downstream of phosphatidylinositol 3kinase during muscle differentiation. The American journal of pathology. 2008;172(6):1529-41. Epub 2008/05/07. doi: 10.2353/ajpath.2008.070429. PubMed PMID: 18458092; PubMed Central PMCID: PMC2408414.

34. Kobayashi M, Li L, Iwamoto N, Nakajima-Takagi Y, Kaneko H, Nakayama Y, et al. The antioxidant defense system Keap1-Nrf2 comprises a multiple sensing mechanism for responding to a wide range of chemical compounds. Molecular and cellular biology. 2009;29(2):493-502. Epub 2008/11/13. doi: 10.1128/MCB.01080-08. PubMed PMID: 19001094; PubMed Central PMCID: PMC2612520. 
35. Jung Y, Kim H, Min SH, Rhee SG, Jeong W. Dynein light chain LC8 negatively regulates NF-kappaB through the redox-dependent interaction with IkappaBalpha. The Journal of biological chemistry. 2008;283(35):23863-71. Epub 2008/06/27. doi: 10.1074/jbc.M803072200. PubMed PMID: 18579519; PubMed Central PMCID: PMC3259766.

36. Li $\mathrm{Q}$, Engelhardt JF. Interleukin-1beta induction of NFkappaB is partially regulated by $\mathrm{H} 2 \mathrm{O} 2-$ mediated activation of NFkappaB-inducing kinase. The Journal of biological chemistry. 2006;281(3):1495-505. Epub 2005/11/16. doi: 10.1074/jbc.M511153200. PubMed PMID: 16286467.

37. Mathieu J, Zhang Z, Zhou W, Wang AJ, Heddleston JM, Pinna CM, et al. HIF induces human embryonic stem cell markers in cancer cells. Cancer Res. 2011;71(13):4640-52. doi: 10.1158/00085472.CAN-10-3320. PubMed PMID: 21712410; PubMed Central PMCID: PMCPMC3129496.

38. Lee KE, Simon MC. From stem cells to cancer stem cells: HIF takes the stage. Curr Opin Cell Biol. 2012;24(2):232-5. doi: 10.1016/j.ceb.2012.01.005. PubMed PMID: 22296771.

39. Karin M. NF-kappaB as a critical link between inflammation and cancer. Cold Spring Harb Perspect Biol. 2009;1(5):a000141. doi: 10.1101/cshperspect.a000141. PubMed PMID: 20066113; PubMed Central PMCID: PMCPMC2773649.

40. Iliopoulos D, Hirsch HA, Wang G, Struhl K. Inducible formation of breast cancer stem cells and their dynamic equilibrium with non-stem cancer cells via IL6 secretion. Proc Natl Acad Sci U S A. 2011;108(4):1397-402. doi: 10.1073/pnas.1018898108. PubMed PMID: 21220315; PubMed Central PMCID: PMCPMC3029760.

41. Korkaya H, Liu S, Wicha MS. Regulation of cancer stem cells by cytokine networks: attacking cancer's inflammatory roots. Clin Cancer Res. 2011;17(19):6125-9. doi: 10.1158/1078-0432.CCR-102743. PubMed PMID: 21685479 ; PubMed Central PMCID: PMCPMC3312242.

42. Jang J, Wang Y, Kim HS, Lalli MA, Kosik KS. Nrf2, a regulator of the proteasome, controls selfrenewal and pluripotency in human embryonic stem cells. Stem cells. 2014;32(10):2616-25. Epub 2014/06/05. doi: 10.1002/stem.1764. PubMed PMID: 24895273; PubMed Central PMCID: PMC4165656.

43. Katajisto P, Dohla J, Chaffer CL, Pentinmikko N, Marjanovic N, Iqbal S, et al. Stem cells. Asymmetric apportioning of aged mitochondria between daughter cells is required for stemness. Science. 2015;348(6232):340-3. Epub 2015/04/04. doi: 10.1126/science.1260384. PubMed PMID: 25837514; PubMed Central PMCID: PMC4405120.

44. Liochev $\mathrm{SI}$, Fridovich I. The effects of superoxide dismutase on $\mathrm{H} 2 \mathrm{O} 2$ formation. Free radical biology \& medicine. 2007;42(10):1465-9. Epub 2007/04/24. doi: 10.1016/j.freeradbiomed.2007.02.015. PubMed PMID: 17448892.

45. Zhao Q, Wang J, Levichkin IV, Stasinopoulos S, Ryan MT, Hoogenraad NJ. A mitochondrial specific stress response in mammalian cells. Embo J. 2002;21(17):4411-9. PubMed PMID: 12198143.

46. Benedetti C, Haynes CM, Yang Y, Harding HP, Ron D. Ubiquitin-like protein 5 positively regulates chaperone gene expression in the mitochondrial unfolded protein response. Genetics. 2006;174(1):22939. PubMed PMID: 16816413.

47. Haynes CM, Petrova K, Benedetti C, Yang Y, Ron D. ClpP mediates activation of a mitochondrial unfolded protein response in C. elegans. Dev Cell. 2007;13(4):467-80. PubMed PMID: 17925224.

48. Nargund AM, Fiorese CJ, Pellegrino MW, Deng P, Haynes CM. Mitochondrial and nuclear accumulation of the transcription factor ATFS-1 promotes OXPHOS recovery during the UPR(mt). Mol Cell. 2015;58(1):123-33. doi: 10.1016/j.molcel.2015.02.008. PubMed PMID: 25773600; PubMed Central PMCID: PMC4385436.

49. Liu Z, Butow RA. Mitochondrial retrograde signaling. Annu Rev Genet. 2006;40:159-85. doi: 10.1146/annurev.genet.40.110405.090613. PubMed PMID: 16771627.

50. Jovaisaite V, Mouchiroud L, Auwerx J. The mitochondrial unfolded protein response, a conserved stress response pathway with implications in health and disease. The Journal of experimental 
biology. 2014;217(Pt 1):137-43. doi: 10.1242/jeb.090738. PubMed PMID: 24353213; PubMed Central PMCID: PMC3867496.

51. Bennett CF, Vander Wende H, Simko M, Klum S, Barfield S, Choi H, et al. Activation of the mitochondrial unfolded protein response does not predict longevity in Caenorhabditis elegans. Nature communications. 2014;5:3483. doi: 10.1038/ncomms4483. PubMed PMID: 24662282; PubMed Central PMCID: PMC3984390.

52. Mouchiroud L, Houtkooper RH, Moullan N, Katsyuba E, Ryu D, Canto C, et al. The NAD(+)/Sirtuin Pathway Modulates Longevity through Activation of Mitochondrial UPR and FOXO Signaling. Cell. 2013;154(2):430-41. doi: 10.1016/j.cell.2013.06.016. PubMed PMID: 23870130; PubMed Central PMCID: PMC3753670.

53. Papa L, Germain D. SirT3 regulates the mitochondrial unfolded protein response. Mol Cell Biol. 2014;34(4):699-710. doi: 10.1128/MCB.01337-13. PubMed PMID: 24324009; PubMed Central PMCID: PMC3911493.

54. Kim HS, Patel K, Muldoon-Jacobs K, Bisht KS, Aykin-Burns N, Pennington JD, et al. SIRT3 is a mitochondria-localized tumor suppressor required for maintenance of mitochondrial integrity and metabolism during stress. Cancer Cell. 2010;17(1):41-52. doi: 10.1016/j.ccr.2009.11.023. PubMed PMID: 20129246; PubMed Central PMCID: PMCPMC3711519.

55. Jin SM, Youle RJ. The accumulation of misfolded proteins in the mitochondrial matrix is sensed by PINK1 to induce PARK2/Parkin-mediated mitophagy of polarized mitochondria. Autophagy. 2013;9(11):1750-7. doi: 10.4161/auto.26122. PubMed PMID: 24149988; PubMed Central PMCID: PMC4028334.

56. Papa L, Germain D. Estrogen receptor mediates a distinct mitochondrial unfolded protein response. J Cell Sci. 2011;124(Pt 9):1396-402. doi: 10.1242/jcs.078220. PubMed PMID: 21486948; PubMed Central PMCID: PMC3078808.

57. Rimmele $\mathrm{P}$, Liang $\mathrm{R}$, Bigarella $\mathrm{CL}$, Kocabas $\mathrm{F}$, Xie J, Serasinghe $\mathrm{MN}$, et al. Mitochondrial metabolism in hematopoietic stem cells requires functional FOXO3. EMBO Rep. 2015;16(9):1164-76. doi: 10.15252/embr.201439704. PubMed PMID: 26209246; PubMed Central PMCID: PMC4576984.

58. Mohrin M, Shin J, Liu Y, Brown K, Luo H, Xi Y, et al. Stem cell aging. A mitochondrial UPRmediated metabolic checkpoint regulates hematopoietic stem cell aging. Science. 2015;347(6228):13747. doi: 10.1126/science.aaa2361. PubMed PMID: 25792330; PubMed Central PMCID: PMC4447312.

59. Semenza GL. Hypoxia-inducible factors in physiology and medicine. Cell. 2012;148(3):399-408. doi: 10.1016/j.cell.2012.01.021. PubMed PMID: 22304911; PubMed Central PMCID: PMCPMC3437543.

60. Majmundar AJ, Wong WJ, Simon MC. Hypoxia-inducible factors and the response to hypoxic stress. Mol Cell. 2010;40(2):294-309. doi: 10.1016/j.molcel.2010.09.022. PubMed PMID: 20965423; PubMed Central PMCID: PMCPMC3143508.

61. Chandel NS, McClintock DS, Feliciano CE, Wood TM, Melendez JA, Rodriguez AM, et al. Reactive oxygen species generated at mitochondrial complex III stabilize hypoxia-inducible factor-1alpha during hypoxia: a mechanism of O2 sensing. J Biol Chem. 2000;275(33):25130-8. doi: 10.1074/jbc.M001914200. PubMed PMID: 10833514.

62. Zou X, Santa-Maria C, O'Brien J, Gius D, Zhu Y. MnSOD acetylation and dys-regulation, due to loss of SIRT3 activity, promotes a Luminal B-like breast carcinogenic permissive phenotype. Antioxid Redox Signal. 2016. doi: 10.1089/ars.2016.6641. PubMed PMID: 26935174.

63. Zhu Y, Park SH, Ozden O, Kim HS, Jiang H, Vassilopoulos A, et al. Exploring the electrostatic repulsion model in the role of Sirt3 in directing MnSOD acetylation status and enzymatic activity. Free Radic Biol Med. 2012;53(4):828-33. doi: 10.1016/j.freeradbiomed.2012.06.020. PubMed PMID: $22732184 ;$ PubMed Central PMCID: PMCPMC3418453. 
64. Ozden O, Park SH, Kim HS, Jiang H, Coleman MC, Spitz DR, et al. Acetylation of MnSOD directs enzymatic activity responding to cellular nutrient status or oxidative stress. Aging (Albany NY). 2011;3(2):102-7. PubMed PMID: 21386137; PubMed Central PMCID: PMCPMC3082006.

65. Tao R, Coleman MC, Pennington JD, Ozden O, Park SH, Jiang $H$, et al. Sirt3-mediated deacetylation of evolutionarily conserved lysine 122 regulates MnSOD activity in response to stress. Mol Cell. 2010;40(6):893-904. doi: 10.1016/j.molcel.2010.12.013. PubMed PMID: 21172655; PubMed Central PMCID: PMCPMC3266626.

66. Jin C, Qin L, Shi Y, Candas D, Fan M, Lu CL, et al. CDK4-mediated MnSOD activation and mitochondrial homeostasis in radioadaptive protection. Free Radic Biol Med. 2015;81:77-87. doi: 10.1016/j.freeradbiomed.2014.12.026. PubMed PMID: 25578653; PubMed Central PMCID: PMCPMC4359946.

67. Ansenberger-Fricano K, Ganini D, Mao M, Chatterjee S, Dallas S, Mason RP, et al. The peroxidase activity of mitochondrial superoxide dismutase. Free Radic Biol Med. 2013;54:116-24. doi: 10.1016/j.freeradbiomed.2012.08.573. PubMed PMID: 22982047; PubMed Central PMCID: PMCPMC4155036.

68. Ganini D, Petrovich RM, Edwards LL, Mason RP. Iron incorporation into MnSOD A (bacterial Mndependent superoxide dismutase) leads to the formation of a peroxidase/catalase implicated in oxidative damage to bacteria. Biochim Biophys Acta. 2015;1850(9):1795-805. doi: 10.1016/j.bbagen.2015.05.006. PubMed PMID: 25964067; PubMed Central PMCID: PMCPMC4516619.

\section{Figure Legends}

Figure 1 - Summary of our hypothesis that the mechanism of electron flow through the ETC leading to ATP or $\mathrm{H}_{2} \mathrm{O}_{2}$ production determines cell plasticity and phenotypic decisions.

Figure 2 - Summary of our hypothesis that mitochondria exists in different functional states characterized by the efficiencies of ATP (state I) or $\mathrm{H}_{2} \mathrm{O}_{2}$ (state II) production. It is further hypothesized that while mitochondrial ATP production in state I sustains differentiation by meeting the energetic demands of specialized cells, $\mathrm{H}_{2} \mathrm{O}_{2}$, produced when mitochondria does not dispose of means to efficiently produce ATP, activates survival programs characterized, for instance, by altered metabolism and HIF signaling. Prolongation of these alterations leads to reprogramming and the appearance of cellular phenotypes amenable to dedifferentiation.

Figure 3 - Example of a superoxide sink in mitochondria. Superoxide is the major source of mitochondrial $\mathrm{H}_{2} \mathrm{O}_{2}$. Once formed, mostly from electrons leaking from complex I and III directly to oxygen, superoxide can react with electron acceptors in mitochondria what regenerates oxygen and restricts $\mathrm{H}_{2} \mathrm{O}_{2}$. Cytochrome $\mathrm{c}$ is an example of a superoxide sink. In fact the reaction of superoxide with oxidized cytochrome c-Fe(III) to generate oxygen and reduced cytochrome c-Fe(II) has been used in analytical biochemistry as an assay to quantify superoxide production in various systems.

Figure 4 - Schematic representation of the mechanisms by which the UPR ${ }^{\mathrm{mt}}$ enhances mitochondrial resilience against high $\mathrm{H}_{2} \mathrm{O}_{2}$ allowing for the simultaneous maintenance of function and production of $\mathrm{H}_{2} \mathrm{O}_{2}$ (state II).

\section{Glossary}


Adenosine triphosphate (ATP): a nucleotide capable of storing chemical energy via phosphate anhydride bonds.

Electron Transport Chain (ETC): Sequence of electron donor/electron accepting complexes that couples electron flow to the formation of the electrochemical potential in mitochondria. The electrochemical potential is used to power ATP synthesis from ADP and inorganic phosphate.

Hypoxia-induced factors (HIFs): transcription factors stabilized under levels of low oxygen availability that activate programs required for cell survival at reduced oxygen availability.

NAD+/FAD (Nicotinamide-adenine dinucleotide and Flavin-adenine dinucleotide): Electron storage units in the cell used as cofactors by oxidase and dehydrogenase enzymes. NADH and $\mathrm{FADH}_{2}$ (the reduced forms of $\mathrm{NAD}^{+}$and $\mathrm{FADH}_{2}$ ) serve as primary electron donors to the ETC.

Nuclear Factor $\mathbf{K B}(\mathbf{N F} \mathbf{K B})$ : a master regulator of transcription activated by inflammatory stimuli recently involved in the acquisition of stemness properties by some cells (e.g. cancer cells).

Nuclear factor-erythroid 2-related factor-2 (Nrf2): a regulator of transcription that responds to oxidative stress and is inhibited by Kelch-like ECH associated protein-1 (Keap-1), a scavenger of Nrf2 that is inhibited by oxidation.

Sirtuins: A family of deacetylase enzymes regulators of cell signaling. Sirtuins are implicated in the control of the aging process, longevity, the metabolism and as tumor suppressors.

Superoxide Dismutase 2 or Manganese superoxide dismutase (SOD2/MnSOD): a mitochondrial, manganese dependent dismutase of superoxide. SOD2 has been proposed to perform an antioxidant function in mitochondria, but more recent literature indicates that a more accurate role of SOD2 is as a source of $\mathrm{H}_{2} \mathrm{O}_{2}$ in mitochondria. In association with enzymes that detoxify $\mathrm{H}_{2} \mathrm{O}_{2}$, SOD2 may work as a component of the mitochondrial antioxidant system.

Reactive Oxygen Species (ROS): Acronym used to define a large number of different molecules that are chemically reactive and contain oxygen. Examples include: Hydrogen Peroxide (oxidant, soluble in aqueous and lipophilic media); superoxide radical (reductant, charged, soluble in aqueous media); peroxynitrous acid/peroxynitrite (oxidant, soluble in aqueous media, diffuses through membranes in the peroxynitrous acid form), and $\mathrm{HOCl}$ (oxidant, soluble in aqueous media, diffuses through membranes).

\section{Box 1: The Roles of ROS and HIF in simulated hypoxia}

Sensing and utilizing oxygen is essential for respiration and the energetic balance of most cells. Variations in oxygen levels occur normally during exercise, tissue growth and wounding and also during deficits in tissue perfusion. When oxygen levels drop the alpha subunits of hypoxiainduced factors are stabilized to form HIF transcription factors ${ }^{[59]}$. HIFs inform the cells of the need to rapidly reform the metabolism, activate survival pathways that normally include a degree of undifferentiation (particularly when hypoxia is prolonged) while also informing the host that increased oxygen supply is needed ${ }^{[59,60]}$. This is done by activating programs in the cells 
that produce ATP independently of oxygen (i.e. glycolysis), and by enhancing the expression of factors that promote angiogenesis (i.e. vascular endothelial growth factor (VEGF)). Because $\mathrm{HIFa}$ is constantly expressed and rapidly degraded in the presence of oxygen, loss of oxygen immediately translate into accumulation of HIFa. HIFa degradation is inhibited by ROS, and in particular by $\mathrm{H}_{2} \mathrm{O}_{2}{ }^{[61]}$. Hence we contend that chronic increases in ROS simulate hypoxia by stabilizing HIFa independently of oxygen abundance thereby uncoupling oxygen sensing from the emergence of (mal)adapted hypoxic cellular phenotypes.

\section{Box 2: SOD2 regulation and activities}

It is becoming increasingly clear that SOD2 is a hub for redox signaling in mitochondria and that its processing of ROS generated in mitochondria broadly affects cellular signaling networks. Hence it is critical to understand how SOD2 works and how it is regulated in mitochondria. Recent studies have demonstrated that SOD2 is posttranslationally regulated for instance by acetylation of key lysine residues that inhibits ${ }^{[62-65]}$ or the phosphorylation of a serine that enhances ${ }^{[66]}$ SOD2 function as a superoxide dismutase. Other studies showed that SOD2 may under specific conditions display a completely different function and work as a peroxidase amplifying oxidative stress ${ }^{[67]}$. Though the mechanisms by which SOD2 is turned into a peroxidase are still incompletely understood recent evidence indicates that structural changes to the protein that facilitate binding to iron are likely involved ${ }^{[68]}$. Hence it is possible that a more complete framework pertaining to how mitochondrial ROS are formed and utilized in signaling will emerge from a better understanding of how SOD2 works and the co-regulators that balance its activities in mitochondria. 


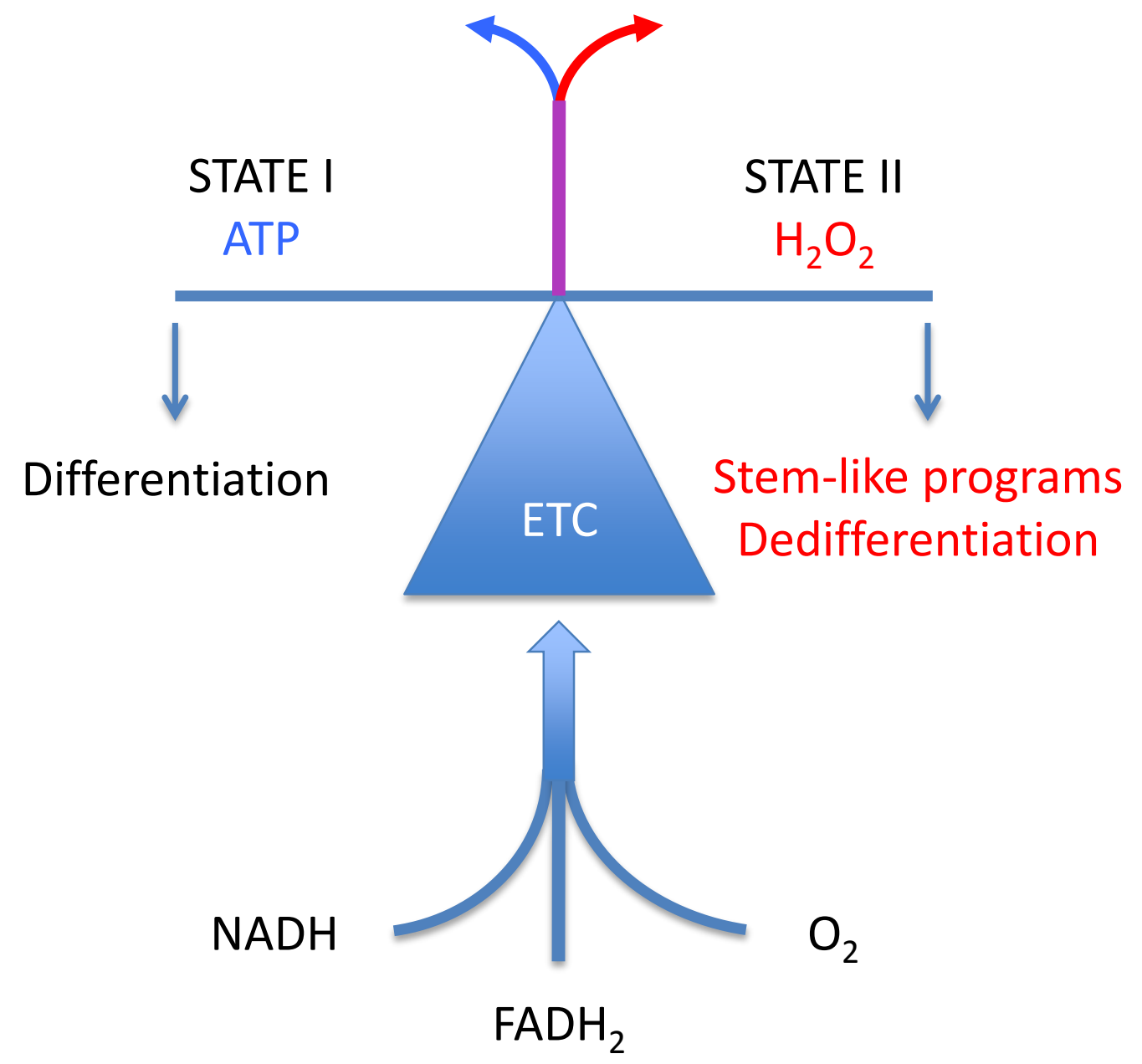

Figure 1 - Summary of our hypothesis that the mechanism of electron flow through the ETC leading to ATP or $\mathrm{H}_{2} \mathrm{O}_{2}$ production determines cell plasticity and phenotypic decisions. 
STATE II

Undifferentiated

$\left(\mathrm{H}_{2} \mathrm{O}_{2}\right.$ high/low energy demand)
STATE I

Differentiated

(ATP high/high energy demand)
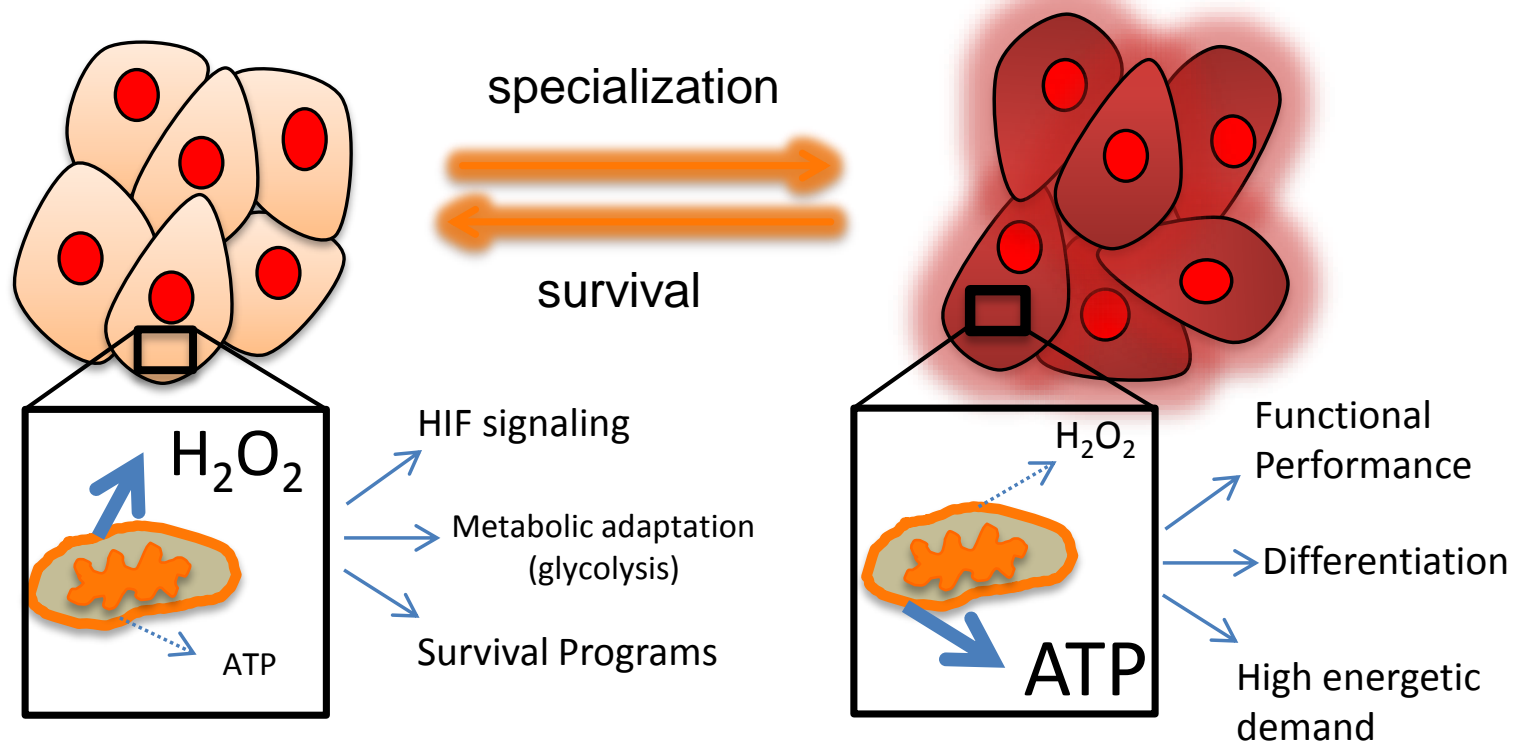


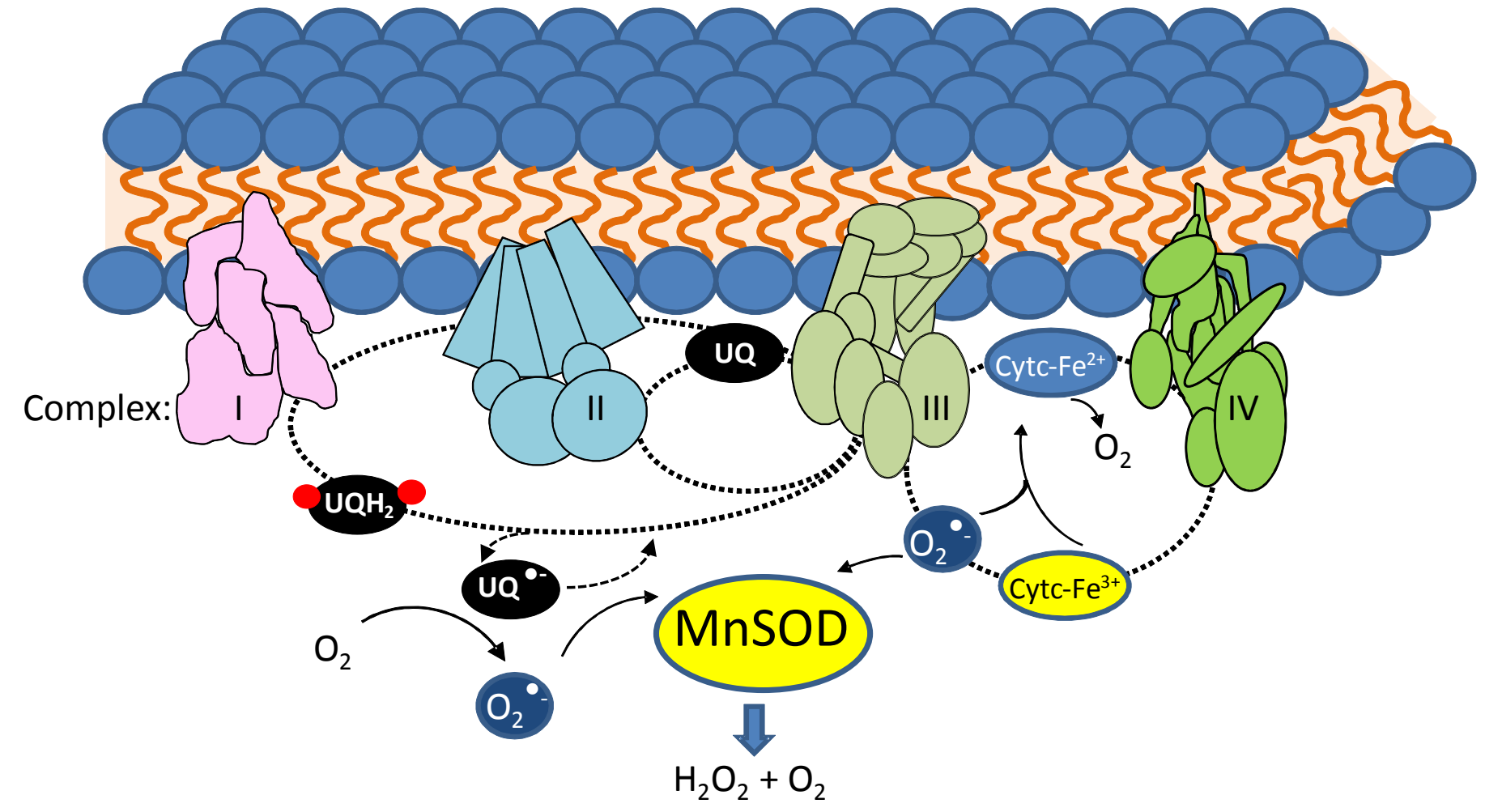




\section{STATE II}

Undifferentiated

$\left(\mathrm{H}_{2} \mathrm{O}_{2}\right.$ high/low energy demand)

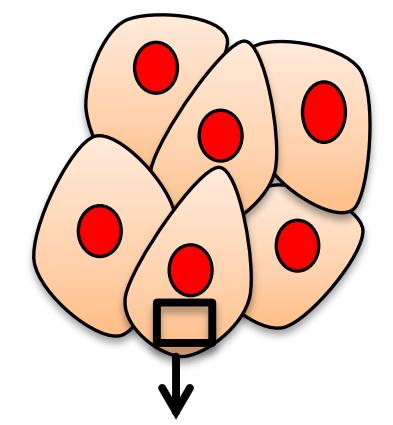

Activation of the UPR ${ }^{m t}$

Differentiation

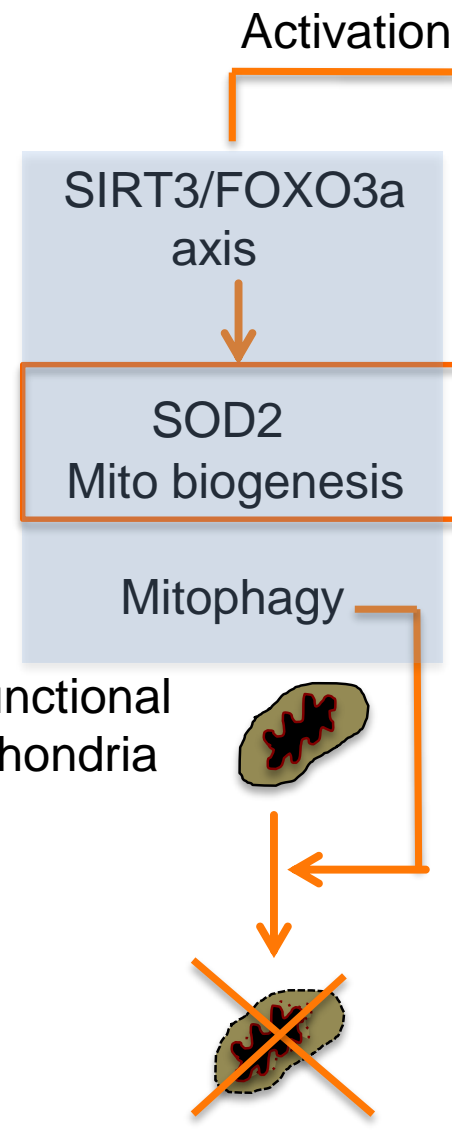

Chaperones

Proteases
CHOP

axis

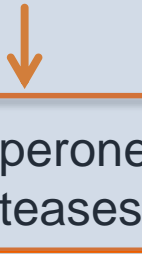

\begin{abstract}
High $\mathrm{H}_{2} \mathrm{O}_{2}$ but
\end{abstract}

Functional

mitochondria

\section{STATE I}

Differentiated

(ATP high/high energy demand)

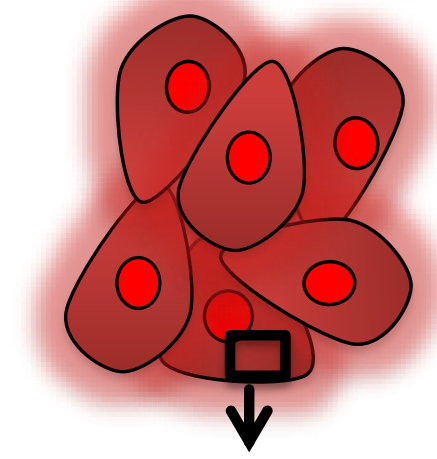

Deactivation of the UPR ${ }^{\mathrm{mt}}$
Dysfunctional mitochondria

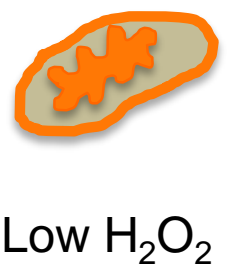

Functional mitochondria 